\title{
Monitoring the 2016 LA County Sand Fire with Multiple Early Detection Systems
}

\author{
Rachel Viola*, Monica Z. Luarca, Emily Kajita, Michael Lim and Bessie Hwang
}

Acute Communicable Disease Control, Los Angeles County Department of Public Health, Los Angeles, CA, USA

\section{Objective}

To detect increases in health complaints resulting from the July 2016 Sand Fire near Santa Clarita, CA using syndromic surveillance and complementary systems.

\section{Introduction}

On July 22, 2016, the Sand Fire began burning in the Santa Clarita Valley of Los Angeles County (LAC), CA. This urban-adjacent wildfire breached the city limits of Santa Clarita (population 180,000). Fueled by record heat and an ongoing exceptional drought, the Sand Fire burned over 40,000 acres in 13 days $^{1}$ and caused a large increase in the air concentration of fine particulate matter ${ }^{2}$. The syndromic surveillance team was tasked with reporting on possible health effects from the fire. Fire, asthma, and heat related data were monitored until the fire was reported as $98 \%$ contained. The team prepared and distributed a daily special summary report to key stakeholders in the LAC Department of Public Health.

\section{Methods}

Emergency department (ED) data were queried for cases related to fire, asthma, cardiac events, eye irritation, heat, and total volume. These queries consisted of key word searches within chief complaint (CC), diagnosis and triage note data fields. Queries were conducted on all participating syndromic EDs in LAC, and also restricted to nine EDs closest to the fire. The resulting line lists were reviewed daily to rule out visits that were unrelated to the Sand Fire. The fire query was refined periodically with additional exclusion terms. Complaints related to asthma were tallied in a second query. In order to assess heat-related ED visits and temperature trends, existing queries and report templates were modified to focus on the nine fire-area EDs. Local temperatures were taken from the Weather Underground website. Complementary systems were also monitored, including over-the-counter medication sales and nurse hotline call data. Trend graphs for hospital admissions and ED visits were produced daily to assess volume from 19 Reddinet participating hospitals. In addition to internal data sources, the South Coast Air Quality Management District website was checked daily to monitor air quality in the Santa Clarita Valley.

\section{Results}

There were 48 syndromic ED patient records with direct mention of the fire in LAC's syndromic hospitals in 13 days. Of these, 26 did not include asthma, and 32 came from the nine hospitals in the Sand Fire region; 32 were identified from the $\mathrm{CC}$, six by diagnosis and ten by triage note. Despite an increase in fire-related visits, overall trends in ED data were not affected; no increase was found for cardiac events, eye irritation, heat-related illness or total volume. Asthma visits increased at the time of the fire, which correlates with a sharp increase in the concentration of fine particulate matter in the Santa Clarita Valley following the start of the fire ${ }^{2}$. However, these increases were no higher than other peaks observed in previous months ${ }^{3}$. No increases in calls to a nurse hotline or over-the-counter medication sales were observed. Among Reddinet hospitals, admissions increased slightly but ED visits remained unchanged.

\section{Conclusions}

For the Sand Fire, ED volume alone was not enough to estimate the subsequent health effects on residents of LAC; instead a specific fire query was needed. Several factors could explain why overall trends were not affected. In a region where air quality is already compromised, it is challenging to distinguish between asthma increases from air pollution from those exacerbated by wildfire smoke. It is also likely that residents heeded warnings about air quality during active fires, thus reducing their outdoor exposure. Although the majority of cases were identified using the $\mathrm{CC}$ field, additional data fields such as triage notes available from some hospitals improve the ability to elicit fire related visits. Regardless of the challenges presented in measuring health effects related to wildfires, syndromic surveillance and complementary systems continue to be the primary tools for near real-time assessments in LAC.

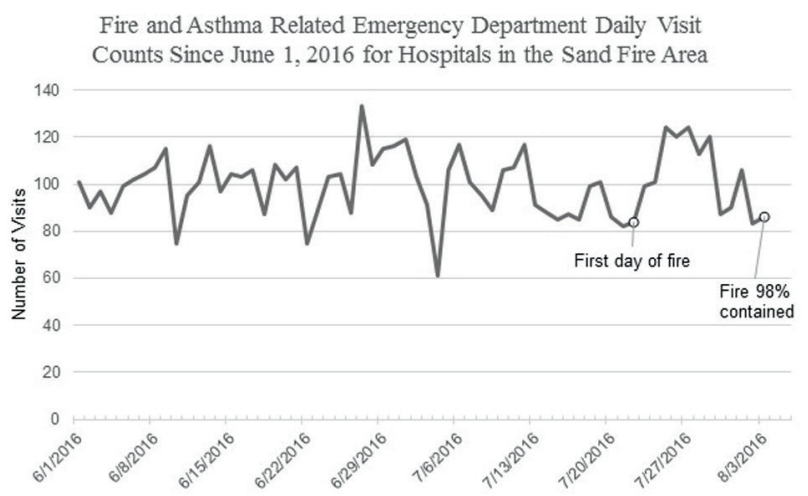

Keywords

syndromic surveillance; disaster; wildfire; early detection

\section{References}

1. Angeles National Forest. Sand Fire [Internet]. [place unknown]: United States Forest Service [updated 2006 Aug 6; cited 2016 Aug 19]. Available from: http://inciweb.nwcg.gov/incident/4878/.

2. Air Quality Data \& Studies [Internet]. Diamond Bar, CA: South Coast Air Quality Management District [cited 2016 Aug 19]. Available from: http://www.aqmd.gov/home/library/air-quality-data-studies.

3. Barboza T. SoCal hit with worst smog in years as hot, stagnant weather brings surge in hospital visits. Los Angeles Times [Internet]. 2016 Aug 11 [cited 2016 Aug 19]; L.A. Now. Available from: http://www. latimes.com/local/lanow/la-me-ln-summer-smog-20160805-snapstory.html.

\section{${ }^{*}$ Rachel Viola}

E-mail: rviola@ph.lacounty.gov 